\title{
Supergravity Null Scissors and Super-Crosses
}

\author{
Bin Chen \\ Physics Division \\ National Center for Theoretical Sciences \\ Hsinchu 300, Taiwan \\ bchen@phys.cts.nthu.edu.tw \\ Chiang-Mei Chen \\ Department of Physics \\ National Taiwan University \\ Taipei 106, Taiwan \\ cmchen@phys.ntu.edu.tw \\ Feng-Li Lin \\ Physics Division \\ National Center for Theoretical Sciences \\ Hsinchu 300, Taiwan \\ fllin@phys.cts.nthu.edu.tw
}

\begin{abstract}
In this paper we construct the supergravity solutions for the orthogonally intersecting null scissors and the fluxed D-strings. We name the latter as the super-crosses according to their shape. It turns out that the smeared solutions are U-dual related to the intersecting $(p, q)$-strings. Their open string properties are also studied. As a by-product, we clarify the supersymmetry conditions of D2-D2 pairs with most generic fluxes.
\end{abstract}

KEYWORDS: Brane in motion. 


\section{Contents}

1. Introduction 1

2. Supersymmetric fluxed D2-D2 pairs and their T-duals 2

3. Supergravity Solutions 5

3.1 From $\mathrm{D} 1 \perp \mathrm{F} 1$ and $(p, q)$-string to KK and boosted D-strings

3.2 Super crosses and null scissors 6

3.3 Decoupling limit? 8

3.4 Wiggled super-crosses and null scissors 9

4. Stability Analysis of Static Fluxed D-String Pair 10

5. Conclusions 11

A. Stability Analysis at 1-loop 12

B. Formula for double T-dual super-cross 14

\section{Introduction}

String theory in the cosmological background is a challenging subject to explore. One essential issue is to understand the spacelike singularity in the context of string theory. One could expect that there should have no singularity due to the limit on the minimal length scale in string theory. Or in other words, the singularities would be resolved. This is a well-known phenomenon in the case of resolution of orbifold and conifold singularities.

A toy solvable model for the above purpose is the so called null brane background discovered by Simon [1]. Many studies on this model have been done [2], especially on the issue of the singularity resolution. Unfortunately the study in [2] indicates that there is an instability to form black hole near the orbifold singularity due to the large blue shift effect. Further study on the nature of the singularity is obscured by the non-linear nature of the closed string theory.

The blue shift effect of the null brane frustrates the original plan of studying the resolution of the cosmic singularities by string theory, however, the mathematical structure of the model is by itself interesting. This motivates people to study the open string analogue of the null brane, the so called null scissors as proposed and studied in [3] and [4] (see also [5] and [6]), and to understand the nature of the singularity without facing the non-linearity associated with gravity. Null scissor is the configuration of two moving intersecting Dstrings with the intersection moving at the speed of light. Surprisingly, a classical instability 
of the null scissor is identified as the failure for the charged string to catch up the motion of the intersecting point even though the open string theory preserves $1 / 4$ supersymmetry. However, authors in [3] tend to believe that the singularity will be resolved though the detailed dynamics is not completely clear yet.

As pointed out in [4] the null scissor configurations are closely related to the D-brane objects with nonzero fluxes, which preserves unexpected supersymmetry and can be understood as the U-duals of the known supersymmetric intersecting branes. Of among them, the supertube [7, 9, 10] is the most important and inspiring one. The U-duals of the supergravity solution of the supertube has been studied in the recent paper [15].

The supersymmetry of the null scissors is also coming out of surprise as for the one of supertube. However, there is yet no supergravity solution for the null scissor and its Uduals. The authors of [15] commented that the boosted string, which is one component of the null scissor, can be obtained by T-dualizing their solution for D2-D0-F1 configuration. We will see that the full solution of the null scissor will arise from the U-dual of the well-known $D 1 \perp F 1$ configuration.

In this paper we will fulfill the supergravity construction of the orthogonally intersecting null scissors and its U-duals. One of its U-duals is the orthogonally intersecting fluxed D-strings, which is named as the "super-cross" for the sake of its shape. The key point of the construction is the realization of the boosted D-string as the double T-dual of the $(p, q)$-string. From this fact we can relate the orthogonally intersecting null scissors to the S-dual of the well-known D1 $\perp$ F1 configuration [11.

This paper is organized as follows. In the next section we analyze the supersymmetry conditions of the D2-D2 pairs with the most general E and B fluxes on them. The technical details of checking the open string partition functions are also given in the Appendix A. We then T-dualize the supersymmetry conditions to the ones in IIB string for the null scissors and super-crosses. In section 3, we construct the supergravity solutions of the null scissors and super-crosses. The distinction between the KK D-string and the boosted D-string is given in 3.1, the supergravity solutions of the super-crosses are constructed from the Sdual of $\mathrm{D} 1 \perp \mathrm{F} 1$ in 3.2 and are shown to be double T-dual to the orthogonally intersecting null scissors. The double T-duality rule is given in the Appendix B. The non-existence of the decoupling limit of the solutions is commented in 3.3. Generalization to the wiggled solutions is discussed in 3.4. In the section 4 we discuss the classical stability issue of the super-cross. Finally we conclude our paper in section 5 .

\section{Supersymmetric fluxed D2-D2 pairs and their T-duals}

In this section we generalize the supersymmetry fluxed D2-brane pair studied in 㘬 to the more general case where the electric fluxes on different D2-branes can point to different directions. After T-duality, we can arrive not just the null scissors but also the one with fluxes on D-strings so that the speed of the intersecting point can be subliminal.

Following 画, the supersymmetry constraints for the flat D2-D2 pair are

$$
\Gamma^{(1)} \epsilon=\epsilon
$$




$$
\begin{aligned}
& \Gamma^{(2)} \epsilon=\epsilon, \\
& {\left[\Gamma^{(1)}, \Gamma^{(2)}\right] \epsilon=0,}
\end{aligned}
$$

where $\epsilon$ is the 10-dim. Killing spinor and $\Gamma^{(i)}$ 's are the Hermitian traceless product structure given by the $\kappa$-symmetry [8], and takes the following form in the static gauge

$$
\Gamma^{(i)}=\frac{1}{\mathcal{L}_{i}}\left[\Gamma_{012}+\left(\Gamma_{2} F_{01}^{(i)}+\Gamma_{0} F_{12}^{(i)}+\Gamma_{1} F_{20}^{(i)}\right) \Gamma_{11}\right]
$$

Here $\Gamma_{m}$ 's, with $m=0 \ldots 9$, are the constant 10-dim. Dirac matrices such that $\left\{\Gamma_{m}, \Gamma_{n}\right\}=$ $2 \eta_{m n}$, and $\Gamma_{11}=\Gamma_{0} \cdots \Gamma_{9}$. Also, $\Gamma_{m \ldots k} \equiv \Gamma_{m} \cdots \Gamma_{k}$. The $\mathcal{L}_{i}$ is the DBI lagrangian for each D2. For simplicity, we have set the constant $2 \pi \ell_{s}^{2}$ in front of the generalized Born-Infeld field $F$ to unity.

The difference of our analysis from the one in [⿶凵⿴囗十 is that we will turn on the generic background field

$$
F^{(i)}=E_{i} d x^{2} \wedge d x^{0}+G_{i} d x^{1} \wedge d x^{0}+B_{i} d x^{2} \wedge d x^{1}
$$

This gives

$$
\mathcal{L}_{i}=\sqrt{1-\left(E_{i}^{2}+G_{i}^{2}-B_{i}^{2}\right)} .
$$

From (2.1) to (2.3) we can turn the compatibility condition (2.3) into the the additional traceless Hermitian product structure

$$
\Gamma^{\prime}=\frac{\left(E_{1} G_{2}-E_{2} G_{1}\right) \Gamma_{0}+\left(E_{1} B_{2}-E_{2} B_{1}\right) \Gamma_{2}+\left(G_{1} B_{2}-G_{2} B_{1}\right) \Gamma_{1}}{\mathcal{L}_{2}-\mathcal{L}_{1}}
$$

satisfying

$$
\Gamma^{\prime} \epsilon=\epsilon
$$

and

$$
\left[\Gamma^{\prime}, \Gamma^{(i)}\right]=0
$$

so that the new supersymmetry conditions (2.1), (2.2) and (2.8) could be diagonalized simultaneously. From (2.8) we shall require

$$
\Gamma^{\prime 2}=1
$$

which gives

$$
G_{2}^{2}=\frac{-\left(E_{1} B_{2}-B_{1} E_{2}\right)^{2}-\left(E_{1} \mp E_{2}\right)^{2}+\left(B_{1} \mp B_{2}\right)^{2}}{\mathcal{L}_{1}^{2}}
$$

if we set $G_{1}=0$ by using the freedom of rotational symmetry.

As known the above condition is only necessary but may not be sufficient. We can pin down the relative sign by setting $G_{2}=0$ and compare it with the results in [4], then we arrive

$$
\Gamma^{\prime} \epsilon= \pm \epsilon, \quad G_{2}= \pm \sqrt{\frac{-\left(E_{1} B_{2}-B_{1} E_{2}\right)^{2}-\left(E_{1}-E_{2}\right)^{2}+\left(B_{1}-B_{2}\right)^{2}}{\mathcal{L}_{1}^{2}}} .
$$


Moreover, we need to impose the following relation

$$
E_{1} E_{2}-B_{1} B_{2}-1 \leq 0
$$

to ensure the consistent D2-D2 supersymmetry condition.

Similarly we can consider the supersymmetry conditions for the D2-antiD2 with various gauge fluxes. Now, the supersymmetry conditions are then

$$
\Gamma^{(1)} \epsilon=\epsilon, \quad \Gamma^{(2)} \epsilon=-\epsilon
$$

and $\left[\Gamma^{(1)}, \Gamma^{(2)}\right] \epsilon=0$. The corresponding additional Hermitian product structure to the compatibility condition is

$$
\tilde{\Gamma}=-\frac{\left(E_{1} G_{2}-E_{2} G_{1}\right) \Gamma_{0}+\left(E_{1} B_{2}-E_{2} B_{1}\right) \Gamma_{2}+\left(G_{1} B_{2}-G_{2} B_{1}\right) \Gamma_{1}}{\mathcal{L}_{2}+\mathcal{L}_{1}} .
$$

The condition $\tilde{\Gamma}^{2}=1$ will yield the same supersymmetry condition $(2.12)$, i.e. with $\Gamma^{\prime}$ replaced by $\tilde{\Gamma}$, but with a different consistency condition

$$
E_{1} E_{2}-B_{1} B_{2}-1 \geq 0
$$

As a consistent check of these supersymmetry conditions, one could calculate the 1-loop partition function of open string between D2s. In Appendix A, using lightcone boundary state formalism [12], we show that the partition functions vanish in both the case of D2D2 and D2-antiD2 under corresponding supersymmetry conditions.

Before ending this section we will look into the T-dual D-string configurations ${ }^{1}$. Our main interest is focused on the case $G_{1}=0$. If we perform the T-duality along the $x^{1}$ direction, then we will get two D-strings spanning an angle $\theta$, with one of them being static and carrying E-flux $e_{1}$, and the other being moving with the normal speed $\beta_{2}$ and carrying E-flux $e_{2}$, the supersymmetry condition (2.12) then becomes

$$
-\beta_{2}^{2}\left(1-e_{1}^{2}\right)+\sin ^{2} \theta=e_{1}^{2}+e_{2}^{2}\left(1-\beta_{2}^{2}\right)-2 e_{1} e_{2} \sqrt{1-\beta_{2}^{2}} \cos \theta .
$$

However, if we perform the T-duality along the $x^{2}$ direction, we will instead obtain two moving D-strings spanning an angle $\theta$, with only one of them carrying flux $e_{2}$, and the supersymmetry condition (2.12) becomes

$$
-e_{2}^{2}\left(1-\beta_{1}^{2}\right)\left(1-\beta_{2}^{2}\right)+\sin ^{2} \theta=\beta_{1}^{2}+\beta_{2}^{2}-2 \beta_{1} \beta_{2} \cos \theta .
$$

One of the simple configurations from (2.17) is to set $\beta_{2}=0$, and we get the static fluxed scissors constrained by

$$
e_{1}^{2}+e_{2}^{2}-2 e_{1} e_{2} \cos \theta=\sin ^{2} \theta .
$$

The super-crosses are the ones with $\theta=\pi / 2$, which will be the starting point to obtain the supergravity description in the next section.

\footnotetext{
${ }^{1}$ In the appendix of 4 one can find the T-dual transformation between a single fluxed D2 and a single fluxed moving D-string at an angle.
} 
On the other hand, by setting $e_{2}=0$ of $(2.18)$ we get the moving null scissors with the intersecting point moving at the speed of light, which can be seen by re-writing the supersymmetry condition into

$$
\left(\beta_{1} \csc \theta+\beta_{2} \cot \theta\right)^{2}+\beta_{2}^{2}=1
$$

From the similarity of the form (2.19) and (2.20), it is clear that we can transmute super null scissors and super-crosses by double T-duality along the orthogonal directions.

\section{Supergravity Solutions}

In this section we would like to construct the supergravity solutions for the null scissors and super-crosses by using various $\mathrm{T}$ and $\mathrm{S}$ dualities. Moreover, they can be related to each other by the S-duality and the doubled T-duality, namely, T-dualizing twice along two orthogonal directions. We summarize the relations in a duality map. In the appendix $\mathrm{B}$, we list the formulae for the double T-duality.

\subsection{From $\mathbf{D} 1 \perp \mathbf{F} 1$ and $(p, q)$-string to $\mathrm{KK}$ and boosted D-strings}

Before going to null scissors and super-crosses, we will start with the simpler configurations, namely, the $\mathrm{D} 1 \perp \mathrm{F} 1$ and $(\mathrm{p}, \mathrm{q})$-string; both are supersymmetric configurations. After the double T-duality of both configurations, we will get the D-string with Kalzua-Klein(KK) momentum and the boosted D-string respectively.

It is known that a fundamental string orthogonally intersecting with Dp-brane preserves $1 / 4$ supersymmetry [11]. Moreover, the supergravity solution can be written down explicitly according to the intersecting rules for the harmonic functions. For $p=1$ with D-string lying on $x$-axis and F-string on $y$-axis, which will be our starting point for constructing the other solutions, the configuration is

$$
\begin{aligned}
& d s^{2}=e^{\frac{\phi}{2}}\left[-H_{f}^{-\frac{3}{4}} H_{d}^{-\frac{3}{4}} d t^{2}+H_{f}^{\frac{1}{4}} H_{d}^{-\frac{3}{4}} d x^{2}+H_{f}^{-\frac{3}{4}} H_{d}^{\frac{1}{4}} d y^{2}+H_{f}^{\frac{1}{4}} H_{d}^{\frac{1}{4}}\left(d r^{2}+r^{2} d \Omega_{6}^{2}\right)\right], \\
& e^{2 \phi}=H_{f}^{-1} H_{d}, \quad B_{[2]}=-\left(H_{f}^{-1}-1\right) d t \wedge d y, \quad C_{[2]}=-\left(H_{d}^{-1}-1\right) d t \wedge d x
\end{aligned}
$$

where the harmonic functions are given by $H_{f}(r)=1+Q_{f} / r^{5}$ and $H_{d}(r)=1+Q_{d} / r^{5}$ with parameters $Q_{f}, Q_{d}$ characterizing the charges of F-string and D-string.

After double T-duality ${ }^{2}$ as given in the Appendix B, which changes the location of $\mathrm{D}$-string from $x$-axis to $y$-axis and converts the $\mathrm{F} 1$ charge into a KK moment on the $y$ direction, we get the D-string with KK momentum as expected, the solution is

$$
\begin{aligned}
d s^{2} & =H_{d}^{-\frac{1}{2}}\left[-d t^{2}+\left(H_{f}-1\right)(d t+d y)^{2}+d y^{2}\right]+H_{d}^{\frac{1}{2}}\left(d x^{2}+d r^{2}+r^{2} d \Omega_{6}^{2}\right), \\
& =H_{d}^{-\frac{1}{2}}\left[-d u d v+\left(H_{f}-1\right) d u^{2}\right]+H_{d}^{\frac{1}{2}}\left(d x^{2}+d r^{2}+r^{2} d \Omega_{6}^{2}\right) \\
e^{2 \phi} & =H_{d}, \quad C_{[2]}=-\left(H_{d}^{-1}-1\right) d t \wedge d y,
\end{aligned}
$$

\footnotetext{
${ }^{2}$ The order of these two T-dualities is immaterial.
} 
where the light cone coordinates are defined as $u=t+y$ and $v=t-y$. For simplicity, we will call this configuration KK D-string.

On the other hand, if we start with a $(p, q)$-string located along the $x$-axis and smeared along the $y$-axis,

$$
\begin{aligned}
d s^{2} & =e^{\frac{\phi}{2}}\left[H^{-\frac{3}{4}}\left(-d t^{2}+d x^{2}\right)+H^{\frac{1}{4}}\left(d y^{2}+d r^{2}+r^{2} d \Omega_{6}^{2}\right)\right], \\
e^{2 \phi} & =\frac{\left(p^{2}+q^{2} H\right)^{2}}{\left(p^{2}+q^{2}\right)^{2} H}, \quad C_{[0]}=-\frac{p q(1-H)}{p^{2}+q^{2} H}, \\
B_{[2]} & =-p\left(H^{-1}-1\right) d t \wedge d x, \quad C_{[2]}=-q\left(H^{-1}-1\right) d t \wedge d x,
\end{aligned}
$$

where the harmonic function $H=1+Q / r^{5}$. This solution can be obtained by applying S-duality to either the F-string or D-string solution.

Performing the double T-duality of this configuration, we obtain a D-string boosted along the transverse direction, and the solution looks as

$$
\begin{aligned}
& d s^{2}=e^{-\phi}\left\{-e^{2 \phi} H^{-1} d t^{2}+H\left[p\left(H^{-1}-1\right) d t-d x\right]^{2}+d y^{2}\right\}+e^{\phi}\left(d r^{2}+r^{2} d \Omega_{6}^{2}\right), \\
& e^{2 \phi}=\frac{p^{2}+q^{2} H}{p^{2}+q^{2}}, \quad C_{[2]}=-q e^{-2 \phi}(1-H)\left(d t+\frac{p}{p^{2}+q^{2}} d x\right) \wedge d y .
\end{aligned}
$$

The location of D-string and the direction of the boost are clearly shown in the expressions of potential and metric. To distinguish from the previous configuration, we will call this the boosted D-string. Similarly, we can construct the boosted F-string configuration via S-duality.

As an interesting exercise, we can get the $(p, q)$ string configuration as the T-dual of $D 2-D 0-F 1$ which is given in the (5.8) of [15] by T-dualizing the boosted D-string. To be explicit, the T-dual of (5.8) in [15] is

$$
\begin{aligned}
d s^{2} & =-f^{\frac{1}{2}} g^{-1} d t^{2}+f^{\frac{1}{2}}\left[d x^{2}+d y^{2}-\left(g^{-1}-1\right)(\cos \theta d x+\sin \theta d y)^{2}+d r^{2}+r^{2} d \Omega_{6}^{2}\right], \\
e^{2 \phi} & =f^{2} g^{-1}, \quad C_{[0]}=\left(f^{-1}-1\right) \sinh \alpha \\
B_{[2]} & =\left(g^{-1}-1\right) \tanh \alpha(\cos \theta d x+\sin \theta d t) \wedge d y=-\sinh \alpha C_{[2]},
\end{aligned}
$$

where

$$
f=1+\frac{Q^{\prime}}{r^{5}}, \quad g=1+\frac{Q^{\prime} \cosh ^{2} \alpha}{r^{5}} .
$$

If $\theta=0$ this configuration is reduced to the $(p, q)$-string configuration (3.3) if we identify

$$
p=\tanh \alpha, \quad q=-\frac{1}{\cosh \alpha}, \quad Q=Q^{\prime} \cosh ^{2} \alpha .
$$

Note that $p^{2}+q^{2}=1$ satisfies the charge quantization condition of the $(p, q)$-string.

\subsection{Super crosses and null scissors}

From the discussions in the previous subsection, it is now clear that if we would like to have the null scissor configuration which is the superposition of two boosted D-strings, we need to start with the configuration of two orthogonally $(p, q)$-string, and then perform the double T-duality. 
The general supersymmetric configuration for the intersecting $(p, q)$-string at any angle is not known, however, the one with orthogonal intersection can be obtained by S-dualizing the $\mathrm{D} 1 \perp \mathrm{F} 1$ configuration (3.1), and we obtain the super-cross configuration as follows:

$$
\begin{aligned}
d s^{2} & =e^{\frac{\phi}{2}}\left[-H_{f}^{-\frac{3}{4}} H_{d}^{-\frac{3}{4}} d t^{2}+H_{f}^{\frac{1}{4}} H_{d}^{-\frac{3}{4}} d x^{2}+H_{f}^{-\frac{3}{4}} H_{d}^{\frac{1}{4}} d y^{2}+H_{f}^{\frac{1}{4}} H_{d}^{\frac{1}{4}}\left(d r^{2}+r^{2} d \Omega_{6}^{2}\right)\right], \\
e^{2 \phi} & =\frac{\left(p_{1}^{2} H_{d}+p_{2}^{2} H_{f}\right)^{2}}{\left(p_{1}^{2}+p_{2}^{2}\right)^{2} H_{f} H_{d}}, \quad C_{[0]}=\frac{p_{1} p_{2}\left(H_{d}-H_{f}\right)}{p_{1}^{2} H_{d}+p_{2}^{2} H_{f}}, \\
B_{[2]} & =-d t \wedge\left[p_{2}\left(H_{d}^{-1}-1\right) d x+p_{1}\left(H_{f}^{-1}-1\right) d y\right], \\
C_{[2]} & =-d t \wedge\left[p_{1}\left(H_{d}^{-1}-1\right) d x-p_{2}\left(H_{f}^{-1}-1\right) d y\right] .
\end{aligned}
$$

Note that the harmonic functions $H_{f, d}$ now becomes $1+\frac{\alpha_{p} Q_{f, d}}{r^{5}}$ with $\alpha_{p}=\sqrt{p_{1}^{2}+p_{2}^{2}}$ to have correct $(p, q)$-string bound state charges.

This solution describes the smeared configuration of two orthogonally intersecting $(p, q)$-strings, namely $(p 1, p 2) \perp(-p 2, p 1)$. However, interestingly it can also be considered as a composed configuration of two superposed $\mathrm{D} 1 \perp \mathrm{F} 1$ bound states, namely $p_{1}(\mathrm{D} 1 \perp \mathrm{F} 1) \oplus$ $p_{2}(\mathrm{~F} 1 \perp \mathrm{D} 1)$. Since both configurations carry the same charges we are not able to distinguish them. The evidence for these twofold descriptions can be explored by considering the following special limits: The super-cross reduces to $\mathrm{D} 1 \perp \mathrm{F} 1$ by simply setting either $p_{1}=1, p_{2}=0$ or $p_{1}=0, p_{2}=1$ and to the $(p, q)$-strings by assuming the $H_{f}=1, H_{d}=H, p_{2}=p, p_{1}=q$. This degeneracy is understood as a coincidence due to the initial symmetric configuration (3.1).

Now we can obtain the orthogonally intersecting null scissor configuration by performing the double T-duality on (3.8), we then get

$$
\begin{aligned}
d s^{2} & =e^{-\phi}\left\{-e^{2 \phi} H_{f}^{-1} H_{d}^{-1} d t^{2}+H_{d}\left[p_{2}\left(H_{d}^{-1}-1\right) d t-d x\right]^{2}+H_{f}\left[p_{1}\left(H_{f}^{-1}-1\right) d t-d y\right]^{2}\right\} \\
& +e^{\phi}\left(d r^{2}+r^{2} d \Omega_{6}^{2}\right), \\
e^{2 \phi} & =\frac{p_{1}^{2} H_{d}+p_{2}^{2} H_{f}}{p_{1}^{2}+p_{2}^{2}} \\
C_{[2]} & =e^{-2 \phi}\left\{-d t \wedge\left[p_{2}\left(1-H_{f}\right) d x+p_{1}\left(1-H_{d}\right) d y\right]+\frac{p_{1} p_{2}}{p_{1}^{2}+p_{2}^{2}}\left(H_{d}-H_{f}\right) d x \wedge d y\right\} .
\end{aligned}
$$

In comparison with the single boosted D-string configuration (3.4) the null scissor configuration looks much like the "superposition" of the two orthogonally intersecting boosted Dstrings as one should expect. Moreover, due to the degeneracy between $(p 1, p 2) \perp(-p 2, p 1)$ and $p_{1}(\mathrm{D} 1 \perp \mathrm{F} 1) \oplus p_{2}(\mathrm{~F} 1 \perp \mathrm{D} 1)$ the configuration (3.9) should also describe two orthogonally intersecting KK D-strings.

As shown in section 2, the open string analysis suggested that the E-fluxes on the super-cross should satisfy the relation $e_{1}^{2}+e_{2}^{2}=1$ for orthogonal intersection. From the NS-NS 2-form $B_{[2]}$ of configuration (3.8) we find that the E-fluxes per $(p, q)$-string bound state charge is just $p_{1}$ and $p_{2}$ respectively, so that the supersymmetric condition requires

$$
p_{1}^{2}+p_{2}^{2}=1
$$


This condition is the same as the one imposing on the parameters of the S-duality to recover the charge quantization, namely, the $S O(2)$ subgroup of $S L(2, R)$ is selected [13]. On the other hand, if the supersymmetry condition is for the E-fluxes per D-string charge, which is either $-p_{1} / p_{2}$ or $p_{2} / p_{1}$, it will be in conflict with the charge quantization condition (3.10).

For the case $p_{1}^{2}+p_{2}^{2}=1$, in which the parameters can be chosen as $p_{1}=\cos \omega, p_{2}=$ $\sin \omega$, the solution has a very simple form

$$
\begin{aligned}
d s^{2} & =e^{-\phi}\left[-d t^{2}+\left(H_{d}-1\right)(\sin \omega d t+d x)^{2}+\left(H_{f}-1\right)(\cos \omega d t+d y)^{2}+d x^{2}+d y^{2}\right] \\
& +e^{\phi}\left(d r^{2}+r^{2} d \Omega_{6}^{2}\right), \\
e^{2 \phi} & =H_{d} \cos ^{2} \omega+H_{f} \sin ^{2} \omega, \\
C_{[2]} & =e^{-2 \phi}\left\{-d t \wedge\left[\sin \omega\left(1-H_{f}\right) d x+\cos \omega\left(1-H_{d}\right) d y\right]+\sin \omega \cos \omega\left(H_{d}-H_{f}\right) d x \wedge d y\right\} .
\end{aligned}
$$

Furthermore, for the more special case $H_{f}=H_{d}=f$, the above configuration is reduced to a more suggestive form for null scissor

$$
\begin{aligned}
d s^{2} & =f^{-\frac{1}{2}}\left\{-d t^{2}+(f-1)\left[(\sin \omega d t+d x)^{2}+(\cos \omega d t+d y)^{2}\right]+d x^{2}+d y^{2}\right\} \\
& +f^{\frac{1}{2}}\left(d r^{2}+r^{2} d \Omega_{6}^{2}\right), \\
e^{2 \phi} & =f, \quad C_{[2]}=-\left(f^{-1}-1\right) d t \wedge(\sin \omega d x+\cos \omega d y) .
\end{aligned}
$$

In the appropriate gauge choice of potentials, one can easily see that there are momenta both along $x$ - and $y$-axes respectively which can not be "mingled" by a rotation.

Up to now we learn that different fluxed or boosted configurations are related by the T-duality, S-duality or particular reductions, we summarize these relations in a duality map in the Figure 1.

All the configurations in the above duality map are only for the ones related to the orthogonally intersecting branes, it is interesting to construct the general supergravity solution for the null scissors with an angle less than $\pi / 2$. It is easy to see that these configurations cannot be obtained from the $\mathrm{D} 1 \perp \mathrm{F} 1$ by the above S-duality and double T-duality. Even the double T-duality along the different $x-y$ axes is just introduce a trivial rotation on the S-duality parameters, we think that one should start with the nonorthogonally intersecting supersymmetric D1/F1 configuration in order to get the generic null scissors. We leave this construction for the future interest.

\subsection{Decoupling limit?}

After getting the supergravity solutions for the null scissors and the super-crosses, we would like to see that if there is any dual description of Super Yang-Mills theory by taking some decoupling limit. If yes, it could be possible for us to understand the semi-classical instability issue of the null scissors [3, 4] from the dual gravity side.

Unfortunately, it is easy to see from the solutions (3.11) or (3.8) that there exists no such limit. The reason is as following: In order to have a sensible decoupling limit as $\alpha^{\prime} \rightarrow 0$, it requires that $e^{\phi}$ scale as the $\left(\alpha^{\prime}\right)^{0}$ and the metric scale as $\alpha^{\prime}$, however, from the metric, we found that it is not possible to choose the scaling behavior for $H_{f}, H_{d}$ and $r$ 


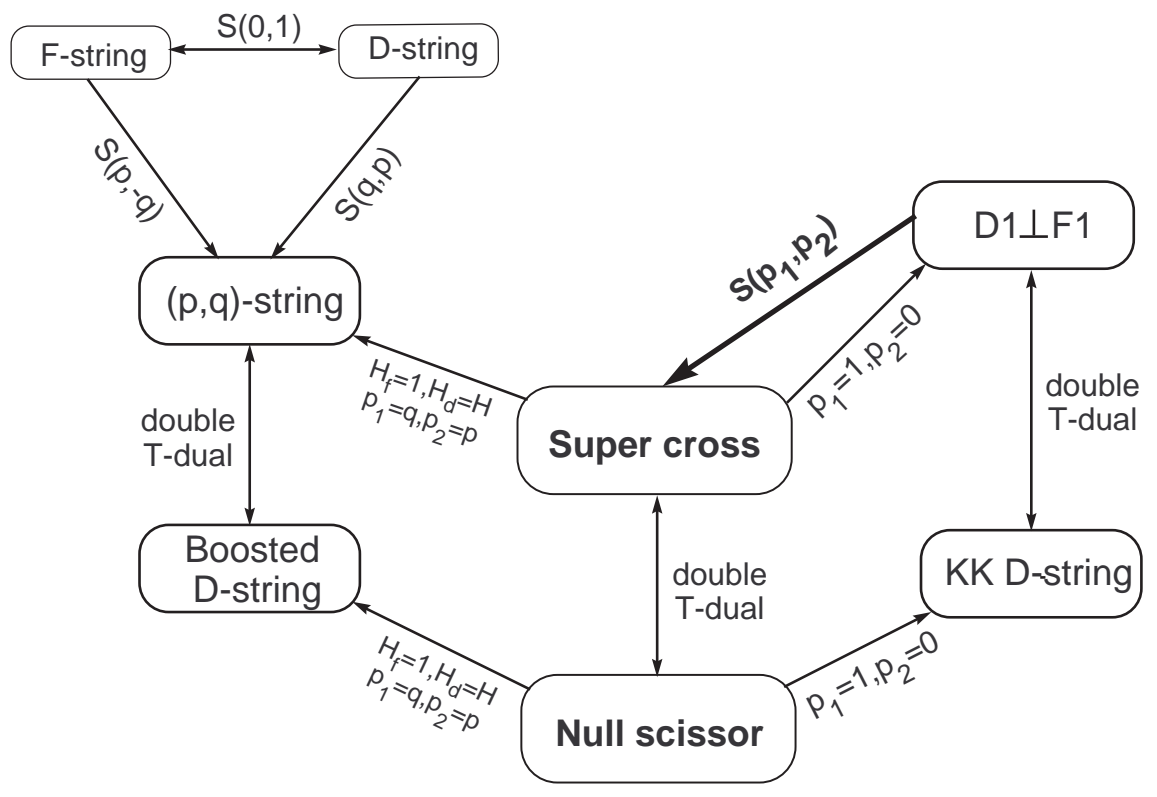

Figure 1: Duality Map

and to keep $g_{Y M}$ fixed so that the metric will scale as $\alpha^{\prime}$. In fact, this is already the case for the configuration of $\mathrm{D} 1 \perp \mathrm{F} 1$.

On the other hand, since there is a possibility to have critical E-field for the supercrosses, one may wonder if there exits a NCOS limit [16]. However, the charged string hanging between the un-fluxed and the fluxed D-strings makes the critical field limit problematic since the two D-strings are not parallel so that the charged string can not be polarized to have zero tension. Moreover, as mentioned, our supergravity solution goes back to the $\mathrm{D} 1 \perp \mathrm{F} 1$ as we takes $p_{1}=0,1$, and we cannot obtain the critical E-flux case from the solution.

\subsection{Wiggled super-crosses and null scissors}

The super-crosses and null scissors can be straightforwardly generalized to a wiggled version. An easy way is starting from an oscillating string [14, 15], a generalization of the KK string which has a transverse profile described by an arbitrary function $\mathbf{F}(u)$,

$$
\begin{aligned}
d s^{2} & =H_{1}^{-1}(\mathbf{z}, u)\left[-d u d v+K(\mathbf{z}, u) d u^{2}+2 A_{i}(\mathbf{z}, u) d z^{i} d u\right]+d x^{2}+d \mathbf{z}^{2}, \\
e^{2 \phi} & =H_{1}^{-1}(\mathbf{z}, u), \\
B_{[2]} & =\frac{1}{2}\left(H_{1}^{-1}(\mathbf{z}, u)-1\right) d u \wedge d v+H_{1}^{-1}(\mathbf{z}, u) A_{i}(\mathbf{z}, u) d u \wedge d z^{i},
\end{aligned}
$$

where the functions are defined as

$$
\begin{aligned}
H_{1}(\mathbf{z}, u) & =1+\frac{Q_{1}}{|\mathbf{z}-\mathbf{F}(u)|^{5}}, \quad H_{2}(\mathbf{z}, u)=1+\frac{Q_{2}}{|\mathbf{z}-\mathbf{F}(u)|^{5}}, \\
K(\mathbf{z}, u) & =\left(H_{1}-1\right)\left(\partial_{u} \mathbf{F}\right)^{2}+\left(H_{2}-1\right), \quad A_{i}(\mathbf{z}, u)=-\left(1-H_{1}\right) \partial_{u} F_{i} .
\end{aligned}
$$


Then all interesting solutions can be constructed at every step of the following approaches.

$$
\begin{aligned}
& \text { Oscillating F-string } \stackrel{S(0,1)}{\longrightarrow} \quad \text { Oscillating D-string } \quad \stackrel{T^{2}}{\longrightarrow} \text { Wiggled D1 } \perp \text { F1 } \\
& S \stackrel{\left(p_{1}, p_{2}\right)}{\longrightarrow} \quad \text { Wiggled Super crosses } \stackrel{T^{2}}{\longrightarrow} \text { Wiggled Null Scissors }
\end{aligned}
$$

Therefore, the wiggled $\mathrm{D} 1 \perp \mathrm{F} 1$ solution is

$$
\begin{aligned}
d s^{2} & =H_{2}^{\prime-1} H_{1}^{-\frac{1}{2}}\left[-\left(d t-A_{i} d z^{i}\right)^{2}+H_{2}^{\prime} d x^{2}+H_{1} d y^{2}\right]+H_{1}^{\frac{1}{2}} d \mathbf{z}^{2} \\
e^{2 \phi} & =H_{2}^{\prime-1} H_{1}, \quad B_{[2]}=-\left(H_{2}^{\prime-1}-1\right) d t \wedge d y-H_{2}^{\prime-1} A_{i} d y \wedge d z^{i}, \\
C_{[2]} & =-\left(H_{1}^{-1}-1\right) d t \wedge d x+H_{1}^{-1} A_{i} d x \wedge d z^{i}
\end{aligned}
$$

where $H_{2}^{\prime}=K+1$.

From this configuration one can perform the S-duality and the double T-dualities as done in the previous subsection to obtain the wiggled super-crosses and null scissors, the procedures are straightforward and the final tedious results are omitted.

\section{Stability Analysis of Static Fluxed D-String Pair}

In this section, we will use the classical Super Yang-Mills theory (SYM) to study the motion of the charged string hanging between the two D-strings.

Start with the equation of motion for the adjoint scalar in the $(1+1) \mathrm{U}(2) \mathrm{SYM}$, that is

$$
D_{a} D^{a} \Phi^{(i)}+\frac{1}{2}\left[\Phi^{(j)},\left[\Phi^{(i)}, \Phi^{(j)}\right]\right]=0
$$

where the $D^{a}=\partial^{a}+i\left[A^{a}, \cdot\right]$ is the covariant derivative.

In order to understand the effect of the electric field on the D-string to the motion of the charged string, we choose the simplest supersymmetric background with the following vevs,

$$
\Phi^{(2)}=\frac{V}{2}\left(\tau_{0}+\tau_{3}\right), \quad A_{1}=\frac{e t}{2}\left(\tau_{0}-\tau_{3}\right),
$$

where the static gauge is chosen so that $A_{0}=0, \Phi^{0}=t, \Phi^{1}=x$, and

$$
V \equiv x \tan \theta+\frac{\beta t}{\cos \theta}
$$

Furthermore, the supersymmetry condition requires

$$
e=\sqrt{\frac{\sin ^{2} \theta-\beta^{2}}{1-\beta^{2}}} .
$$

As mentioned, this configuration describes a pair of D-strings, one lies on the $x$-axis, and the other of nonzero constant E-flux moves with speed $\beta$ in the direction with an angle relative to $x$-axis. This configuration solves the equation of motion but break the gauge symmetry to $U(1) \times U(1)$. 
We introduce the transverse perturbation represented by a charged string in the background of (4.2) as

$$
\delta \Phi^{(\perp)}=\left(\begin{array}{cc}
0 & \phi_{+} \\
\phi_{-} & 0
\end{array}\right),
$$

and the equation of motion for the charged string perturbation is reduced to

$$
\left\{-\partial_{t}^{2}+\partial_{x}^{2} \mp 2 i e t \partial_{x}-(e t)^{2}-\frac{1}{2} V^{2}\right\} \phi_{ \pm}=0,
$$

or to a more physically transparent form as

$$
\left\{p_{t}^{2}-\left(p_{x} \pm A_{x}\right)^{2}-\frac{1}{2} V^{2}\right\} \phi_{ \pm}=0
$$

where $p_{t}=i \partial_{t}, p_{x}=-i \partial_{x}$ and $A_{x}=F_{t x} t=e t$. This describes a pair of oppositely charged particles subjected to a constant electric field and a simple-harmonic-like potential.

If $\beta=0$, the configuration reduces to the static fluxed scissors with $e_{1}=\sin \theta$ and $e_{2}=0$ of (2.19). For $\theta=\pi / 2$ this is just the special case of the super-cross. In these cases, (4.7) just describes a relativistic simple harmonic oscillator subjected to the constant electric field. As known in the non-relativistic case, this configuration is localized and thus it is stable. The stability of the relativistic case should remain if the quantum Schwinger effect of pair production by the E-field is neglected. On the other hand, if $\beta \neq 0$ the potential $V$ is both space and time dependent and (4.7) is hard to solve, it is then not easy to tell the stability in lack of the explicit solution.

We then conclude that the static fluxed scissors should be classically stable. This raises some issue of concern about the stability in the T-dual related configurations. As we found in this paper, the super-cross and null scissor are related by double T-duality and both are related to the fluxed D2-brane pair by T-duality. However, the fluxed D2-brane and the super-cross are classically stable but the null scissor is not [3, 4] although all are supersymmetric and have zero 1-loop result. These facts indicate that the T-duality and the classical stability are not commutative. Though that, we expect that quantum effect will comes into play as the total energy goes high so that the net effect will yield a stable configuration as indicated by the 1-loop result.

\section{Conclusions}

In this paper we have constructed the supergravity configurations for the orthogonally intersecting null scissors and super-crosses. Also we have found a duality map between different intersecting string configurations. As emphasized in the introduction the key point in the construction is the realization of the boosted D-string as the double T-dual of the $(p, q)$-string. The more general supergravity null scissor configurations with arbitrary intersecting angle are expected to be constructed along the same line although one needs to first obtain the more general starting configuration other than the D1 $\perp$ F1.

One of our original motivation for the supergravity solution is to understand the stability issue of the null scissors from the dual gravity side. Unfortunately there exists no 
sensible decoupling limit for these supergravity configurations. It is not clear at all why such obstacle exist for the dual description.

One can also follow the open string spectrum analysis for the null scissor as done in [3] and do different T-duality on the fluxed D2-brane pair to obtain the one for the supercross or static intersecting fluxed D-string. The resulting on-shell condition is complicated and it is hard to extract any conclusive result from that. However, we notice that in the analysis of [3] the oscillator modes in one of the worldvolume direction are suppressed so that the center of mass momentum of the charged string is not dependent on the oscillator modes. It may be difficult to justify the suppression of these oscillator modes as the total energy goes high. This might be a loop hole to the stability issue and the full picture of the resolution of the open string singularity remains as a challenge.

\section{Acknowledgements}

B.C would like to thank the support of Prof. Kiwoon Choi on his stay in KAIST, and also thank the hospitality of KIAS during his visit. The work of CMC is supported by National Science Council and the CosPA project. FLL acknowledges the hospitality of the Lorentz Center where this project was inspired.

\section{A. Stability Analysis at 1-loop}

In this Appendix we will calculate the stringy 1-loop partition function and verify the supersymmetry condition, i.e. zero partition function, for the D2-D2 pair configuration discussed in section 2 .

Let's give a short review of the calculation in [9], where the light-cone gauge boundary state formalism has been used[12]. In the light-cone boundary state formalism, the Dpbrane could be taken as the ' $(p+1)$ ' instanton with Euclidean worldvolume which could be related to the ordinary Lorentz worldvolume through double Wick rotation.

The partition function between two parallel brane can be factorized into

$$
Z=Z_{0} Z_{\text {osci }}
$$

where

$$
Z_{0}=\mathcal{L}_{E}\left(F^{(1)}\right) \mathcal{L}_{E}\left(F^{(2)}\right)\left(T r_{v} M_{r e l}-T r_{s} M_{r e l}\right)
$$

Here, we have

$$
M_{\text {rel }}=M_{2}^{T} \cdot M_{1}
$$

with $M$ being the $\mathrm{SO}(8)$ matrix

$$
M_{I J}=\left(\begin{array}{cc}
M_{i j} & \\
& I_{7-p}
\end{array}\right) .
$$

For the generic flux configuration

$$
F=E d x^{1} \wedge d x^{2}+G d x^{3} \wedge d x^{1}+B d x^{2} \wedge d x^{3},
$$




$$
\begin{aligned}
M_{i j}^{v} & =-(1-F)(1+F)^{-1} \\
& =-\frac{1}{\mathcal{L}_{E}^{2}(F)}\left(\begin{array}{ccc}
1+B^{2}-E^{2}-G^{2} & 2(B G-E) & 2(E B+G) \\
2(E+B G) & 1+G^{2}-B^{2}-E^{2} & 2(-B+E G) \\
2(E B-G) & 2(B+E G) & 1+E^{2}-B^{2}-G^{2}
\end{array}\right)
\end{aligned}
$$

and

$$
M_{i j}^{s}=\frac{1}{\mathcal{L}_{E}(F)} \gamma^{12 \cdots 8}\left(\gamma^{123}+E \gamma^{3}+B \gamma^{1}+G \gamma^{2}\right)
$$

where $\mathcal{L}_{E}(F)=\sqrt{1+E^{2}+B^{2}+G^{2}}$.

In order to consider both $\mathrm{D}-\mathrm{D}$ and $\mathrm{D}$-antiD case, we take into account of the rotation in the $(3,4)$ plane. Then the rotation matrix becomes

$$
M=m^{-1}(\phi) \cdot M \cdot m(\phi)
$$

where

$$
m^{v}(\phi)=\left(\begin{array}{ccccc}
1 & & & \\
& 1 & & & \\
& \cos (\phi) & -\sin (\phi) & \\
& \sin (\phi) & \cos (\phi) & \\
& & & I_{4}
\end{array}\right)
$$

in the vector representation and

$$
m^{s}(\phi)=\exp \left(\frac{\phi}{2} \gamma^{34}\right)=\cos \left(\frac{\phi}{2}\right) I+\sin \left(\frac{\phi}{2}\right) \gamma^{34}
$$

in the spinor representation.

From these profiles, we can read out the zero-mode contribution to the partition function:

$$
Z_{0}=\frac{\lambda}{\mathcal{L}_{E}\left(F_{2}\right) \mathcal{L}_{E}\left(F_{1}\right)}-\rho
$$

where $^{3}$

$$
\begin{aligned}
\lambda & =8\left[E_{1} E_{2}+B_{1} B_{2} G_{1} G_{2}+\left(E_{1} E_{2} B_{1} B_{2}+G_{1} G_{2}+E_{1} E_{2} G_{1} G_{2}+B_{1} B_{2}\right) \cos (\phi)\right] \\
& +4\left(1+E_{1}^{2} E_{2}^{2}+B_{1}^{2} B_{2}^{2}+G_{1}^{2} G_{2}^{2}\right)+4 \mathcal{L}_{E}^{2}\left(F_{2}\right) \mathcal{L}_{E}^{2}\left(F_{1}\right)
\end{aligned}
$$

and

$$
\rho=8\left[\left(1+E_{1} E_{2}\right) \cos (\phi)+B_{1} B_{2}+G_{1} G_{2}\right]
$$

To compare with the brane configuration we discussed in the last section, let us do double Wick rotation and set $G_{1}=0$. Then we find that the zero-mode contribution to

\footnotetext{
${ }^{3}$ Here our interests are on the $\mathrm{D}-\mathrm{D}$ and $\mathrm{D}$-antiD brane configuration so we don't put $\cos 2 \phi$ into the relation since $\cos (2 \phi)=1$ in both cases.
} 
the partition function to be

$$
\begin{aligned}
\lambda & =8\left(-E_{1} E_{2}-E_{1} E_{2} B_{1} B_{2} \cos (\phi)+B_{1} B_{2} \cos (\phi)\right) \\
& +4\left(1+E_{1}^{2} E_{2}^{2}+B_{1}^{2} B_{2}^{2}\right)+4 \mathcal{L}_{1}^{2} \mathcal{L}_{2}^{2} \\
\rho & =8\left(\left(1-E_{1} E_{2}\right) \cos (\phi)+B_{1} B_{2}\right)
\end{aligned}
$$

where $\mathcal{L}_{i}$ 's are defined in (2.6) with $G_{1}=0$.

Firstly, let us consider the D-D case where $\phi=0$ and put (2.11) into the relation, then we obtain

$$
Z_{0}=8\left|1-E_{1} E_{2}+B_{1} B_{2}\right|-8\left(1-E_{1} E_{2}+B_{1} B_{2}\right)=0
$$

under the consistency condition (2.13). This confirms the supersymmetry condition found in section 2. Similarly this is true for the D-antiD case ${ }^{4}$ which corresponds to $\phi=\pi$.

\section{B. Formula for double T-dual super-cross}

Let us consider a general T-dual result of the super-cross, starting from the following general type of solution

$$
\begin{aligned}
& d s^{2}=G_{t t} d t^{2}+G_{x x} d x^{2}+G_{y y} d y^{2}+2 G_{x y} d x d y+G_{r r}\left(d r^{2}+r^{2} d \Omega_{6}^{2}\right), \\
& B_{[2]}=B_{t x} d t \wedge d x+B_{t y} d t \wedge d y, \quad C_{[2]}=C_{t x} d t \wedge d x+C_{t y} d t \wedge d y,
\end{aligned}
$$

with non-vanishing axion $C_{[0]}=C_{0}$ and dilaton $\phi_{0}$.

After T-duality along x-axis, we obtain the new configuration as following

$$
\begin{aligned}
d s^{2} & =\left(G_{t t}+\frac{B_{t x}^{2}}{G_{x x}}\right) d t^{2}+\frac{1}{G_{x x}} d x^{2}+\frac{G}{G_{x x}} d y^{2}+2 \frac{B_{t x}}{G_{x x}} d t d x+G_{r r}\left(d r^{2}+r^{2} d \Omega_{6}^{2}\right), \\
e^{2 \phi} & =\frac{e^{2 \phi_{0}}}{G_{x x}}, \quad B_{[2]}=-\frac{G_{x y}}{G_{x x}} d x \wedge d y+\frac{B}{G_{x x}} d t \wedge d y, \\
C_{[1]} & =\left(C_{t x}+C_{0} B_{t x}\right) d t+C_{0} d x, \quad C_{[3]}=\left(-C_{t y}+\frac{C_{t x} G_{x y}}{G_{x x}}\right) d t \wedge d x \wedge d y,
\end{aligned}
$$

where

$$
G \equiv G_{x x} G_{y y}-G_{x y}^{2}, \quad B \equiv B_{t y} G_{x x}-B_{t x} G_{x y} .
$$

Then we take another T-duality on y-axis and arrive

$$
\begin{aligned}
d s^{2}= & \left(G_{t t}+\frac{B^{\prime} B_{t x}+B B_{t y}}{G}\right) d t^{2}+\frac{G_{y y}}{G} d x^{2}+\frac{G_{x x}}{G} d y^{2} \\
& +2 \frac{B^{\prime}}{G} d t d x+2 \frac{B}{G} d t d y+2 \frac{G_{x y}}{G} d x d y+G_{r r}\left(d r^{2}+r^{2} d \Omega_{6}^{2}\right), \\
e^{2 \phi}= & \frac{e^{2 \phi_{0}}}{G}, \quad B_{[2]}=0 \\
C_{[2]}= & -\left(C_{t y}+C_{0} B_{t y}\right) d t \wedge d x+\left(C_{t x}+C_{0} B_{t x}\right) d t \wedge d y+C_{0} d x \wedge d y,
\end{aligned}
$$

where

$$
B^{\prime} \equiv B_{t x} G_{y y}-B_{t y} G_{x y} .
$$

\footnotetext{
${ }^{4}$ One should let $B_{2} \rightarrow-B_{2}$ because the ant-D2 is obtained by flipping D2 so that the direction of $B 2$ is reversed with respect to $B_{1}$.
} 


\section{References}

[1] J. Simon, "The geometry of null rotation identifications", JHEP 0206 (2002) 001, hep-th/0203201.

[2] H. Liu, G. Moore, N. Seiberg, "Strings in a Time-Dependent Orbifold", JHEP 0206 (2002) 045, hep-th/0204168.

H. Liu, G. Moore, N. Seiberg, "Strings in Time-Dependent Orbifolds", JHEP 0210 (2002) 031, hep-th/0206182.

G. T. Horowitz, J. Polchinski, "Instability of Spacelike and Null Orbifold Singularities", Phys. Rev. D66 (2002) 103512, hep-th/0206228.

M. Fabinger, J. McGreevy, "On Smooth Time-Dependent Orbifolds and Null Singularities", hep-th/0206196.

[3] C. Bachas, C. Hull, "Null Brane Intersections", JHEP 0212 (2002) 035, hep-th/0210269.

C. Bachas, "Relativistic String in a Pulse", hep-th/0212217.

[4] R. C. Myers, D. J. Winters, "From D-D Pair to Branes in Motion", hep-th/0211042.

[5] K. Okuyama, "D-Branes on the Null-Brane", hep-th/0211218.

[6] J.-H. Cho, P. Oh, "Supersymmetric Boost on Intersecting D-branes", JHEP 0301 (2003) 046, hep-th/0212009.

[7] D. Mateos, P.K. Townsend, "Supertubes", Phys. Rev. Lett. 87 (2001) 011602, hep-th/0103030.

R. Emparan, D. Mateos, P.K. Townsend, "Supergravity Supertubes", JHEP 0107 (2001) 011, hep-th/0106012.

[8] E. Bergshoeff, R. Kallosh, T. Ortin and G. Papadopoulos, " $\kappa$-Symmetry, Supersymmetry and Intersecting Branes", hep-th/9705054.

[9] D. Mateos, S. Ng and P.K. Townsend, "Tachyons, Supertubes and Brane/Antibrane Systems", hep-th/0112054.

[10] D. Bak and N. Ohta, "Supersymmetric D2-anti-D2 String," Phys. Lett. B527 (2002) 131-141, hep-th/0112034.

Y. Hyakutake and N. Ohta, "Supertubes and Supercurves from M-Ribbons," Phys. Lett. B539 (2002) 153-161, hep-th/0204161.

R.-G. Cai, J.-X. Lu and N. Ohta, "NCOS and D-Branes in Time-Dependent Backgrounds", Phys. Lett. B551 (2003) 178-186, hep-th/0210206.

[11] J. P. Gauntlett, "Intersecting Branes", hep-th/9705011.

[12] M. Green and M. Gutperle, "Light-cone Supersymmetry and D-brane", hep-th/9604091.

[13] J. H. Schwarz, "An SL(2,Z) Multiplet of Type IIB Superstrings", Phys. Lett. B360 (1995) 13-18; Erratum-ibid. B364 (1995) 252, hep-th/9508143.

[14] G. T. Horowitz, A.A. Tseytlin, "A New Class of Exact Solutions in String Theory", Phys. Rev. D51 (1995) 2896-2917, C. G. Callan, Jr., J. M. Maldacena, A. W. Peet, "Extremal Black Holes As Fundamental Strings", Nucl. Phys. B475 (1996) 645-678, hep-th/9510134.

[15] O. Lunin, J. Maldacena, L. Maoz, "Gravity solutions for the D1-D5 system with angular momentum", hep-th/0212210. 
[16] R. Gopakumar, J. Maldacena, S. Minwalla, A. Strominger, "S-Duality and Noncommutative Gauge Theory", JHEP 0006 (2000) 036, hep-th/0005048.

C.-S. Chan, A. Hashimoto, H. Verlinde, "Duality Cascade and Oblique Phases in Non-Commutative Open String Theory", JHEP 0109 (2001) 034, hep-th/0107215. 